\title{
The Essential Burn Unit Handbook: Jeffrey J. Roth, William B. Hughes (eds)
}

\author{
Published by CRC Press, Taylor \& Francis Group, LLC, FL, 2016; ISBN: 978-1-4987-0571-4
}

\author{
Maneesh Singhal $^{1} \cdot$ Shashank Chauhan ${ }^{1}$
}

Received: 6 April 2016 / Accepted: 6 April 2016/Published online: 29 April 2016

(C) Dr. K C Chaudhuri Foundation 2016

Severe burn injury is the highest level of trauma that can be sustained by the human body. Although the burn injury has been sustained by the skin but there is a profound systemic response by the whole body. Burn patient resuscitation, fluid and electrolyte management, wound management, nutrition, dressings, nursing care, are few but important aspects of the comprehensive management of a burn patient.

This handbook is an excellent companion for quick reference for burn patient management. Medical students and residents, both would benefit immensely by this handbook. It is very easy to carry and the contents are easy to find. The authors have produced a very concise and accurate bundle of basic facts and principles and their clinical applications in the management of burn injured patients.

Maneesh Singhal

drmaneesh@gmail.com

Department of Plastic, Reconstructive and Burns Surgery, All India Institute of Medical Sciences, New Delhi 110029, India
A special mention must be given to the fact that the inclusion of a chapter on (nonburn) inpatient wound care has given a holistic preview for the management of a burn patient in the wards by adding management of pressing issues like pressure sores etc.

Accurate diagrammatic illustrations have made up for the lack of clinical photographs. However, we would like to add that in the diagram of finger escharotomies, the correct sequence of finger escharotomies also needs to be added since the radial aspect of index finger and ulnar aspect of thumb are avoided as the initial sites of escharotomy.

The use of tables and flowcharts in the book will make it very easy and convenient for the surgeon to make sound clinical decisions. It is a must have handbook for the whole burn unit, doctors and nurses alike. 